\title{
Network-based approaches for analysis of complex biological systems
}

\author{
Deborah Chasman $^{\mathrm{a}}$, Alireza Fotuhi Siahpirani ${ }^{\mathrm{b}, \mathrm{a}, \mathrm{c}}$, Sushmita Roy ${ }^{\mathrm{a}, \mathrm{c}, \mathrm{b}, *}$ \\ ${ }^{a}$ Wisconsin Institute for Discovery \\ University of Wisconsin-Madison, Madison, WI 53715 \\ ${ }^{b}$ Department of Computer Sciences \\ University of Wisconsin-Madison, Madison, WI 53706 \\ ${ }^{c}$ Department of Biostatistics and Medical Informatics \\ University of Wisconsin-Madison, Madison, WI 53792
}

\begin{abstract}
Cells function and respond to changes in their environment by the coordinated activity of their molecular components, including mRNAs, proteins and metabolites. At the heart of proper cellular function are molecular networks connecting these components to process extra-cellular environmental signals and drive dynamic, context-specific cellular responses. Network-based computational approaches aim to systematically integrate measurements from highthroughput experiments to gain a global understanding of cellular function under changing environmental conditions. We provide an overview of recent methodological developments toward solving two major computational problems within this field in the past two years (2013-2015): network reconstruction and network-based interpretation. Looking forward, we envision development of methods that can predict phenotypes with high accuracy as well as provide biologically plausible mechanistic hypotheses.

Keywords: Computational systems biology, context-specific network, regulatory network, network-based prediction, gene prioritization, interpretation
\end{abstract}

\footnotetext{
${ }^{*}$ Corresponding author

Email address: sroy@biostat.wisc.edu (Sushmita Roy)
} 


\section{Introduction}

Normal and disease cellular states are the integrated outputs of networks acting at multiple levels of regulation, including the pre-transcriptional, transcriptional and post-translational levels [1, 2, 3, 4]. Advances in omic techniques are providing an unprecedented capacity to measure RNA (coding and non-coding), protein, and post-translational modification levels under different biological contexts such as time, cell states, tissues, or organisms $[5,6,7,8]$. Systematic integration of such datasets is essential to identify molecular networks controlling normal and disease states, and, ultimately, predict complex phenotypes from molecular markers $[9,10]$. This review focuses on recent efforts (2013-2015, Supplementary Table 1) in the field of network biology to address two major challenges that have emerged in the era of high-throughput biology (Fig. 1): Network reconstruction, which entails inference of shared and context-specific network connectivity from multiple omic measurements of molecular entities, and Networkbased interpretation of experimental observations to guide further study.

\section{Network reconstruction}

A long-standing problem in systems biology is to infer causal, regulatory connections among genes, proteins and metabolites and to understand how connections change over time, cell types and other conditions. A network provides a natural representation of a complex cellular system with nodes representing the molecular components and edges representing different types of connectivities (Box 1A). The goal of network reconstruction (also referred to as network inference) methods is to infer such connections from genome-scale measurements of genes, proteins and metabolites from multiple conditions (Fig 1A). In this review, we focus on transcriptional networks and briefly discuss extensions to post-transcriptional, signaling and metabolic networks. We first discuss recent work in the widely studied problem of reverse engineering a single genome-wide network from large collections of gene expression data, followed by approaches to infer integrative and context-specific networks, problems of emerging interest.

\section{Regulatory network reconstruction for one condition}

Since the advent of microarrays and more recently RNA-seq to measure genome-wide transcriptomes, expressionbased network inference methods have been popular for inferring regulatory networks among regulatory proteins such as transcription factors (TFs) and signaling proteins and target genes. A regulatory network model has two components (Box 1B): (a) the structure, which specifies the regulators of a target gene, and (b) the regulatory function, encoded as a mathematical function, which describes how individual and combined regulatory inputs specify a target gene's expression. Several different mathematical functions have been proposed to relate regulatory inputs to expression out- 
put including Boolean functions, ordinary differential equations, thermodynamic models, and probabilistic functions and mammals). A key requirement of such approaches is prior knowledge of cis-regulatory elements on DNA sequence specificity of TFs. Experimental approaches to measure sequence specificity of TFs in vitro (e.g. using protein binding microarrays [25]) and in vivo (e.g. using DNase I (open chromatin) [26], ATAC-seq [27] and chromatin state [28]), 
combined with computational modeling [29, 30], could significantly improve the quality of the prior networks for transcriptional regulatory networks.

More complete and accurate networks require measuring and modeling additional levels of transcriptional and post-transcriptional regulation. Accordingly, new methods are being developed to integrate these aspects. For example, using genome-wide miRNA and mRNA levels measurements from cancer samples, several groups have inferred miRNA-target gene networks [31,32]. Osmanbeyoglu et al. [33]* infer latent activity of and relationships between signaling proteins and transcription factors using a new regularized regression approach, integrating both reverse phase protein array levels and gene expression. Metabolic networks also have regulatory roles and interact with transcriptional networks to control the overall state of organisms [34, 35]. Recent availability of large scale metabolomic profiles have fueled parallel development of network-based methods to analyze metabolomic data. We refer the reader to recent reviews on network approaches with metabolomic data $[36,37]$ and integration of these networks with transcriptional networks [38,39]. A direction that can be particularly useful for capturing different levels of regulation is to better model the distribution of the random variables representing the molecular entities, for example, by allowing heterogeneous random variables $[40,41]$.

Inference of a causal network by asserting directionality on edges is another important challenge in network reconstruction. Gene expression data from natural variation populations [42] or single gene perturbations [43] can help assert directed causal connections. To this end, the caPC (covariate-adjusted PC) algorithm [44] infers a partially directed graph by regressing each gene's expression on a set of single-nucleotide polymorphisms (SNPs), and then uses the PC algorithm [45] to infer directionality based on conditional independence tests. Temporal information can also capture causal dependencies and dynamic Bayesian frameworks are particularly promising to address this [46]*. An alternative is to focus on inferring the directed connections among regulators only, using a prior regulatory network as input and inferring the activity of regulators rather than inferring new edges to targets. The biRte method [47] extends Nested Effects Models [48], to infer a directed graph over the regulators based on the identity and expression of their targets. An advantage of this method is that it does not predict regulator activity only based on mRNA levels. These approaches are promising steps toward solving a key challenge of inferring directionality in regulatory networks.

\section{Context-specificity and dynamics in network reconstruction}

The previous section focused on learning a single network from data. Often it is important to infer how networks change between different contexts (e.g., cell types and diseases) or over time. Recently, several methods have been developed to jointly infer networks that each represent a different context. Because information is shared during the inference process, the networks can be used to study shared and context-specific components. 
The vast majority of methods have used Gaussian Graphical models (GGMs) $[49,50]$ that infer undirected "functional" interactions corresponding to direct statistical dependencies (Box 1C). Learning a GGM translates to estimating the non-zero off-diagonal entries in the inverse of the covariance matrix (also called the precision matrix), further imposing regularization terms to encourage sparsity. One approach by Kling et al. [51]* uses an augmented Sparse Inverse Covariance Selection (SICS) approach to jointly estimate one network (precision matrix) for each cancer, each network predicting connections between genes, microRNAs and sequence mutations. This is accomplished by a regularized regression framework with a novel multi-graph prior that encourages similarity between the networks as well as a modular structure within each network. Other approaches incorporate prior information about the relationship between the contexts. Treegl [52] and GNAT [53]* use a known hierarchy over contexts, such as a cell lineage. In Treegl, data are observed at both internal and leaf nodes, while for GNAT, data are available only in the leaf nodes. Aiming to get closer to a directed regulatory network, Ontogenet [54]* infers per-module regulatory programs for multiple cell types in a lineage. This approach is similar to Treegl and GNAT, except that in Ontogenet the covariates of a gene are restricted to annotated regulators such as transcription factors and one estimates regulators for modules rather than individual genes, making this approach amenable to small sample size problems. GNAT and Ontogenet are of particular interest because they demonstrate that these approaches can be used on a large scale.

Context-specific networks have also been examined using supervised learning by training on a gold-standard network extracted from known interaction databases $(e . g .,[55,56])$ that is filtered based on node expression in a specific condition [57]. Park et al. [58] train tissue-specific support vector machines to predict the probability of an edge belonging to a particular interaction type (transcriptional regulation, phosphorylation, protein co-complexes, and posttranslational regulation). Greene et al. [59]* apply a Bayesian data integration approach to learn tissue-specific networks for 144 tissues based on available human gene expression data sets in the Gene Expression Omnibus [60].

A more fine-grained notion of context is time; however, the above approaches are not applicable here because of the lack of sufficient samples per time point. Yosef et al. [61] overlayed time-point specific gene expression on a skeleton regulatory network inferred from sequence-specific motifs and targets. ODE-based models have the finest possible resolution [62], however, learning such models on a genome-scale is intractable and most approaches have focused on few dozen nodes [46]. To overcome the computational complexity while modeling different types of dependencies including temporal dependencies, the Jump3 method [63] uses tree-based models on time-series data and were able to model hundreds of genes.

Of special interest are methods that use single cell data such as single cell RNA-seq [64, 65] or proteomic levels [66] for inferring regulatory networks in cell fate specification problems $[67,68,66]$. The method of Ocone el al. [67]** uses dimensionality reduction techniques and cellular trajectory learning [69] to infer a pseudo time course. 
They then use an ODE-based model to infer a regulatory network for each of the developmental branches. Because these assays can measure hundreds to thousands of cells, each cell providing a simultaneous measurement of multiple molecules at a time, these technologies open up a new avenue of inferring networks per cell type.

In summary, network reconstruction is the problem of inferring regulatory causal connections between molecular nodes in one or multiple conditions. Expression-based network inference methods have been popular because mRNA levels are the most widely available type of data. However, expression alone is not sufficient to learn regulatory networks, and new approaches that either incorporate prior interactions or additional types of data will be important to learn more accurate and complete networks.

\section{Network-based interpretation and prioritization}

Once a network structure is available, powerful computational approaches have been developed to tackle a variety of problems, broadly grouped under "network-based interpretation and prioritization". Here we address three broad classes of methods: integrating gene sets from multiple high-throughput experiments, examining perturbation in networks, and prioritizing genes for follow up experiments. Common to these approaches is to use the network as a "skeleton" to define the universe of possible physical (e.g., protein-protein, protein-DNA) and functional (genetic) relationships among genes (Box 1C).

\section{Integrating gene hits from complementary high-throughput experiments}

Frequently, multiple high-throughput experimental assays such as transcriptomic or proteomic profiling, as well as large-scale functional screening studies [70,71], are used to identify genes important for a specific biological process [72]. Often, the experimental methodologies are complementary and identify only partially overlapping hit gene sets. An important question is if, and how, the genes identified from each assay are related to each other. Network flow methods (Fig 1D) are popular approaches to identify subnetworks integrating input gene sets based on their connectivity on a skeleton network (reviewed by Kim et al. [73]). The key idea is that the subnetwork depicts how information flows between the different hit sets. For example, a subnetwork can link functional screen hits (upstream) to differential mRNA expression (downstream). Network flow methods often (but not exclusively) seek to find a minimal or sparse subnetwork (i.e., using a small number of edges to connect many hits) and vary in the node-, edge- and path-level properties that are inferred for each subnetwork. For example, prize-collecting Steiner trees [74] and maximum flow [72] methods define node- or edge-level properties (e.g., sign, directionality) and define an objective function that prefers a minimal network. In contrast, candidate path-based methods can incorporate more complex path-level properties $[75,76,77,78,79]$, for example, enabling the connection of specific node pairs. 
However, enumerating candidate paths or optimizing over them can be computationally challenging. As in network reconstruction, an emerging theme among information flow methods is to extend existing approaches to jointly infer subnetworks for multiple related contexts [79, 80, 81].

All methods define these network level properties as constraints and solve the constrained optimization method using either: (i) linear (or integer linear) programming (ILP) methods [72, 74, 76, 77, 78, 79], or (ii) statistical inference within a maximum likelihood framework $[75,82,83]$. When considering genome-scale networks, ILP-based methods have the advantage in that they can find globally optimal solutions more efficiently compared to probabilistic approaches. However, probabilistic approaches are also useful at smaller scales as different parameters can be estimated in a data-driven manner.

\section{Interpreting genetic variation}

A network also provides molecular context to interpret genetic variation (Fig 1D), including Single Nucleotide Polymorphisms (SNPs). Some approaches are applicable when there is a known directionality from the perturbation to specific affected targets. PINE (Perturbations in NEtworks) [84] is a probabilistic method to predict which branches of an input parameterized signaling network are affected by genetic variants under specific conditions. If a less detailed input network is sufficient, network flow methods (e.g., minimal set cover) can also be used. For example, in cancer, identifying causal "driver" variants from correlated "passenger" mutations is important. This problem is commonly addressed by minimum set cover approach (recently, $[85,86])$ to distinguish causal driver mutations from passenger' mutations by finding a small set of drivers that can be connected to the targets by paths in a protein-protein network.

If only a list of mutations is available, or if causal directionality from mutations to gene expression is not assumed, one can use module-based methods to find densely connected components of the network that are enriched for highly mutated genes (e.g., [87, 88]). Grouping the mutated or dysregulated genes into modules may make the approach more resistant to noise in the network or data. Recently this concept has been applied in conjunction with expression data to interpret genetic variation in autism [89] and glioblastoma [90].

A limitation of current skeleton-network based approaches is that they typically include only perturbations to coding regions, and cannot be used to interpret regulatory variants, many of which can lie far away from genes [91]. As our ability to identify regulatory variation improves, for example from high-throughput regulatory genomic datasets $[6,5]$, extending network-based methods to incorporate regulatory variants will become increasingly important $[92$, 93]. 


\section{Gene prioritization}

Protein-protein and functional interaction networks are often used to prioritize genes for further experiments

(Fig 1E). Most of these approaches (reviewed by Moreau \& Tranchevant [94]) have either relied on network centrality measures (e.g., connectivity of hub genes, Box 1D, [95]), or, have used an input set of known relevant genes to rank candidates using the concept of guilt by association: genes that are close to input genes (e.g., based on shortest path length, or more global diffusion-based measures [96]) in the skeleton network are likely to have similar function. An increasingly important and exciting new direction is to prioritize genes to efficiently convert one cell type into another, e.g., to reprogram differentiated cells into induced pluripotent cells [97, 98]. CellNet [98]** first infers tissue and cell type-specific networks, and then uses those networks in a Random Forest classification approach to prioritize genes for reprogramming and to quantify similarity between a query sample and a known cell type. A subsequent approach, Mogrify [97], prioritizes genes based on proximity on an input skeleton network and a measure of differential expression, which is informed by the lineage structure of cell types. Both approaches successfully recapitulated known cell-fate specification genes, and also predicted new genes that were validated to be important regulators for establishing a specific fate.

In summary, network-based methods can be powerful for interpretation of high-throughput measurements and for prioritization of experiments. The choice of the skeleton network is important as most approaches rely on interactions from public databases that may not be relevant to the cellular context being modeled. Recent efforts to infer contextspecific interactions may allow these network-based approaches to achieve higher precision [59].

\section{Conclusions}

Network-based computational methods aim to build, interpret and integrate molecular networks and have been important tools for studies of complex responses. In this review, we discussed recent promising approaches for two major problems: network reconstruction and network-based interpretation. Going forward we envision addressing one major challenge: to build network-based causal, predictive models of complex phenotypes. This will require us to construct networks and prioritize experiments in concert, rather than independently, within an integrated computationalexperimental framework that permits multiple iterations of prediction and validation. Novel network-based models that leverage new types of datasets from emerging technologies for measuring RNA [65] and open chromatin [99] for single cells, as well as approaches to perform targeted perturbations (e.g. using CRISPR/Cas9 technologies [100, 101]) will increasingly enable us to infer causal edges and identify the mechanistic, molecular underpinnings of complex traits and diseases. 


\section{Acknowledgments}

SR is supported by a National Science Foundation CAREER award (NSF DBI: 1350677) and a Sloan Foundation research fellowship. This publication was made possible in part by US Environmental Protection Agency grant 83573701. 


\section{Annotated bibliography}

The following papers are of special $(*)$ or outstanding $(* *)$ interest:

Osmanbeyoglu et al, 2014 [33]*: A novel regularized regression framework (affinity regression) for inferring relationships between signaling proteins and transcription factors using protein and mRNA levels. Notably, this approach

\section{Bibliography}

1. Barabasi, A.L., Gulbahce, N., Loscalzo, J.. Network medicine: a network-based approach to human disease. Nature Reviews Genetics 2011;12(1):56-68. URL: http://dx.doi .org/10.1038/nrg2918. doi:10 . $1038 /$ nrg2918. 
2. Bensimon, A., Heck, A.J.R., Aebersold, R.. Mass spectrometry-based proteomics and network biology. Аnпu Rev Biochem 2012;81:379-405. URL: http://dx.doi.org/10.1146/ annurev-biochem-072909-100424. doi:10.1146/annurev-biochem-072909-100424.

3. Ideker, T., Krogan, N.J.. Differential network biology. Mol Syst Biol 2012;8:565. URL: http://dx. doi . org/10.1038/msb.2011.99. doi:10.1038/msb.2011.99.

4. Lee, T.I., Young, R.A.. Transcriptional regulation and its misregulation in disease. Cell 2013;152(6):12371251. doi:http://dx.doi.org/10.1016/j.cell.2013.02.014.

5. , R.E.C., Kundaje, A., Meuleman, W., Ernst, J., Bilenky, M., Yen, A., Heravi-Moussavi, A., Kheradpour, P., Zhang, Z., Wang, J., Ziller, M.J., Amin, V., Whitaker, J.W., Schultz, M.D., Ward, L.D., Sarkar, A., Quon, G., Sandstrom, R.S., Eaton, M.L., Wu, Y.C., Pfenning, A.R., Wang, X., Claussnitzer, M., Liu, Y., Coarfa, C., Harris, R.A., Shoresh, N., Epstein, C.B., Gjoneska, E., Leung, D., Xie, W., Hawkins, R.D., Lister, R., Hong, C., Gascard, P., Mungall, A.J., Moore, R., Chuah, E., Tam, A., Canfield, T.K., Hansen, R.S., Kaul, R., Sabo, P.J., Bansal, M.S., Carles, A., Dixon, J.R., Farh, K.H., Feizi, S., Karlic, R., Kim, A.R., Kulkarni, A., Li, D., Lowdon, R., Elliott, G., Mercer, T.R., Neph, S.J., Onuchic, V., Polak, P., Rajagopal, N., Ray, P., Sallari, R.C., Siebenthall, K.T., Sinnott-Armstrong, N.A., Stevens, M., Thurman, R.E., Wu, J., Zhang, B., Zhou, X., Beaudet, A.E., Boyer, L.A., De Jager, P.L., Farnham, P.J., Fisher, S.J., Haussler, D., Jones, S.J.M., Li, W., Marra, M.A., McManus, M.T., Sunyaev, S., Thomson, J.A., Tlsty, T.D., Tsai, L.H., Wang, W., Waterland, R.A., Zhang, M.Q., Chadwick, L.H., Bernstein, B.E., Costello, J.F., Ecker, J.R., Hirst, M., Meissner, A., Milosavljevic, A., Ren, B., Stamatoyannopoulos, J.A., Wang, T., Kellis, M.. Integrative analysis of 111 reference human epigenomes. Nature 2015;518(7539):317-330. URL: http://dx.doi.org/10.1038/nature14248.doi:10.1038/nature14248.

6. , E.N.C.O.D.E.P.C.. An integrated encyclopedia of dna elements in the human genome. Nature 2012;489(7414):57-74. URL: http://dx.doi.org/10.1038/nature11247. doi:10.1038/ nature11247.

7. Cancer Genome Atlas Research Network, , Weinstein, J.N., Collisson, E.A., Mills, G.B., Shaw, K.R.M., Ozenberger, B.A., Ellrott, K., Shmulevich, I., Sander, C., Stuart, J.M.. The cancer genome atlas pan-cancer analysis project. Nat Genet 2013;45(10):1113-1120. URL: http://dx. doi .org/10 .1038/ng.2764. doi:10.1038/ng.2764. 
8. GTEx Consortium, . The genotype-tissue expression (gtex) project. Nat Genet 2013;45(6):580-585. URL: http://dx.doi.org/10.1038/ng.2653.doi:10.1038/ng.2653.

9. Cho, D.Y., Kim, Y.A., Przytycka, T.M.. Chapter 5: Network biology approach to complex diseases.

16. Werhli, A.V., Husmeier, D.. Reconstructing gene regulatory networks with bayesian networks by combining expression data with multiple sources of prior knowledge. Stat Appl Genet Mol Biol 2007;6:Article15. URL: http://dx.doi.org/10.2202/1544-6115.1282.doi:10.2202/1544-6115.1282. 
17. Mukherjee, S., Speed, T.P.. Network inference using informative priors. Proc Natl Acad Sci U S A 2008;105(38):14313-14318. URL: http://dx.doi.org/10.1073/pnas.0802272105. doi:10 . $1073 /$ pnas.0802272105.

18. Heckerman, D., Chickering, D.M., Meek, C., Rounthwaite, R., Kadie, C.. Dependency networks for collaborative filtering and data visualization. In: Proceedings of the Sixteenth conference on Uncertainty in artificial intelligence. Morgan Kaufmann Publishers Inc.; 2000:264-273.

19. Greenfield, A., Hafemeister, C., Bonneau, R.. Robust data-driven incorporation of prior knowledge into the inference of dynamic regulatory networks. Bioinformatics 2013;29(8):1060-1067. URL: http://dx. doi . org/10.1093/bioinformatics/btt099. doi:10.1093/bioinformatics/btt099.

20. Petralia, F., Wang, P., Yang, J., Tu, Z.. Integrative random forest for gene regulatory network inference. Bioinformatics 2015;31(12):i197-i205. URL: http://dx.doi.org/10.1093/bioinformatics/ btv268. doi:10.1093/bioinformatics/btv268.

21. Huynh-Thu, V.A., Irrthum, A., Wehenkel, L., Geurts, P.. Inferring regulatory networks from expression data using tree-based methods. PLoS One 2010;5(9). URL: http: / / dx . doi . org/10 .1371/ journal . pone.0012776. doi:10.1371/journal.pone.0012776.

22. Vogel, C., Marcotte, E.M.. Insights into the regulation of protein abundance from proteomic and transcriptomic analyses. Nat Rev Genet 2012;13(4):227-232. URL: http: / / dx . doi .org/10 . 1038/nrg3185. doi:10.1038/nrg3185.

23. Arrieta-Ortiz, M.L., Hafemeister, C., Bate, A.R., Chu, T., Greenfield, A., Shuster, B., Barry, S.N., Gallitto, M., Liu, B., Kacmarczyk, T., et al. An experimentally supported model of the bacillus subtilis global transcriptional regulatory network. Molecular systems biology 2015;11(11):839. doi:10 .15252/msb . 20156236.

24. Liao, J.C., Boscolo, R., Yang, Y.L., Tran, L.M., Sabatti, C., Roychowdhury, V.P.. Network component analysis: reconstruction of regulatory signals in biological systems. Proceedings of the National Academy of Sciences 2003;100(26):15522-15527. doi:10.1073/pnas.2136632100.

25. Siggers, T., Gordân, R.. Protein-dna binding: complexities and multi-protein codes. Nucleic Acids Res 2014;42(4):2099-2111. URL: http://dx.doi.org/10.1093/nar/gkt1112. doi:10.1093/nar/ gkt1112. 
26. Vierstra, J., Stamatoyannopoulos, J.A.. Genomic footprinting. Nature methods 2016;13(3):213-221. doi:doi : $10.1038 /$ nmeth. 3768 .

27. Buenrostro, J.D., Giresi, P.G., Zaba, L.C., Chang, H.Y., Greenleaf, W.J.. Transposition of native chromatin

34. Watson, E., MacNeil, L.T., Arda, H.E., Zhu, L.J., Walhout, A.J.M.. Integration of metabolic and gene regulatory networks modulates the c. elegans dietary response. Cell 2013;153(1):253-266. URL: http: //dx.doi.org/10.1016/j.cell.2013.02.050.doi:10.1016/j.cell.2013.02.050.

35. Oliveira, A.P., Dimopoulos, S., Busetto, A.G., Christen, S., Dechant, R., Falter, L., Haghir Chehreghani, M., Jozefczuk, S., Ludwig, C., Rudroff, F., Schulz, J.C., González, A., Soulard, A., Stracka, D., Aebersold, 
R., Buhmann, J.M., Hall, M.N., Peter, M., Sauer, U., Stelling, J.. Inferring causal metabolic signals that regulate the dynamic torc1-dependent transcriptome. Mol Syst Biol 2015;11(4):802.

36. O'Brien, E.J., Monk, J.M., Palsson, B.O.. Using genome-scale models to predict biological capabilities. Cell 2015;161(5):971-987. URL: http://dx.doi.org/10.1016/j.cel1.2015.05.019. doi:10 . $1016 / j . \operatorname{cell} .2015 .05 .019$.

37. King, Z.A., Lloyd, C.J., Feist, A.M., Palsson, B.O.. Next-generation genome-scale models for metabolic engineering. Curr Opin Biotechnol 2015;35:23-29. URL: http://dx. doi . org/10 . 1016/ j . copbio . 2014.12.016. doi:10.1016/j.copbio.2014.12.016.

38. Gonçalves, E., Bucher, J., Ryll, A., Niklas, J., Mauch, K., Klamt, S., Rocha, M., Saez-Rodriguez, J.. Bridging the layers: towards integration of signal transduction, regulation and metabolism into mathematical models. Molecular BioSystems 2013;9(7):1576-1583.

39. Kim, J., Reed, J.L.. Refining metabolic models and accounting for regulatory effects. Current opinion in biotechnology 2014;29:34-38.

40. Chen, S., Witten, D.M., Shojaie, A.. Selection and estimation for mixed graphical models. Biometrika 2014;::asu051.

41. Žitnik, M., Zupan, B.. Data fusion by matrix factorization. Pattern Analysis and Machine Intelligence, IEEE Transactions on 2015;37(1):41-53.

42. Brem, R.B., Kruglyak, L.. The landscape of genetic complexity across 5,700 gene expression traits in yeast. Proceedings of the National Academy of Sciences of the United States of America 2005;102(5):1572-1577.

43. Kemmeren, P., Sameith, K., van de Pasch, L.A., Benschop, J.J., Lenstra, T.L., Margaritis, T., ODuibhir, E., Apweiler, E., van Wageningen, S., Ko, C.W., et al. Large-scale genetic perturbations reveal regulatory networks and an abundance of gene-specific repressors. Cell 2014;157(3):740-752.

44. Gao, B., Cui, Y.. Learning directed acyclic graphical structures with genetical genomics data. Bioinformatics 2015;URL: http://dx.doi.org/10.1093/bioinformatics/btv513. doi:10.1093/ bioinformatics/btv513.

45. Spirtes, P., Glymour, C.N., Scheines, R.. Causation, prediction, and search; vol. 81. MIT Press; 2000. 
46. Oates, C.J., Dondelinger, F., Bayani, N., Korkola, J., Gray, J.W., Mukherjee, S.. Causal network inference using biochemical kinetics. Bioinformatics 2014;30(17):i468-i474. URL: http : / / dx . doi . org/10 . 1093/bioinformatics/btu452. doi:10.1093/bioinformatics/btu452.

47. Fröhlich, H.. biRte: Bayesian inference of context-specific regulator activities and transcriptional networks. Bioinformatics 2015;URL: http://dx.doi.org/10.1093/bioinformatics/btv379. doi:10.1093/bioinformatics/btv379.

48. Markowetz, F., Kostka, D., Troyanskaya, O.G., Spang, R.. Nested effects models for high-dimensional phenotyping screens. Bioinformatics 2007;23(13):i305-i312.

49. Yuan, M., Lin, Y.. Model selection and estimation in the gaussian graphical model. Biometrika 2007;94(1):1935.

50. Friedman, J., Hastie, T., Tibshirani, R.. Sparse inverse covariance estimation with the graphical lasso. Biostatistics 2008;9(3):432-441.

51. Kling, T., Johansson, P., Sanchez, J., Marinescu, V.D., Jörnsten, R., Nelander, S.. Efficient exploration of pan-cancer networks by generalized covariance selection and interactive web content. Nucleic Acids Res 2015;43(15):e98. URL: http://dx.doi.org/10.1093/nar/gkv413. doi:10.1093/nar/gkv413.

52. Parikh, A.P., Curtis, R.E., Kuhn, I., Becker-Weimann, S., Bissell, M., Xing, E.P., Wu, W.. Network analysis of breast cancer progression and reversal using a Tree-Evolving network algorithm. PLoS Comput Biol 2014;10(7):e1003713+. URL: http://dx.doi.org/10.1371/journal.pcbi.1003713. doi:10. 1371 / journal.pcbi.1003713.

53. Pierson, E., GTEX Consortium, , Koller, D., Battle, A., Mostafavi, S., Ardlie, K.G., Getz, G., Wright, F.A., Kellis, M., Volpi, S., Dermitzakis, E.T.. Sharing and specificity of co-expression networks across 35 human tissues. PLoS Comput Biol 2015;11(5):e1004220. URL: http://dx.doi .org/10.1371/journal. pcbi.1004220. doi:10.1371/journal.pcbi.1004220.

54. Jojic, V., Shay, T., Sylvia, K., Zuk, O., Sun, X., Kang, J., Regev, A., Koller, D., Immunological Genome Project Consortium, , Best, A.J., Knell, J., Goldrath, A., Joic, V., Koller, D., Shay, T., Regev, A., Cohen, N., Brennan, P., Brenner, M., Kim, F., Rao, T.N., Wagers, A., Heng, T., Ericson, J., Rothamel, K., Ortiz-Lopez, A., Mathis, D., Benoist, C., Bezman, N.A., Sun, J.C., Min-Oo, G., Kim, C.C., Lanier, L.L., Miller, J., Brown, B., Merad, M., Gautier, E.L., Jakubzick, C., Randolph, G.J., Monach, P., 
Blair, D.A., Dustin, M.L., Shinton, S.A., Hardy, R.R., Laidlaw, D., Collins, J., Gazit, R., Rossi, D.J., Malhotra, N., Sylvia, K., Kang, J., Kreslavsky, T., Fletcher, A., Elpek, K., Bellemarte-Pelletier, A., Malhotra, D., Turley, S.. Identification of transcriptional regulators in the mouse immune system. Nat Immunol 2013;14(6):633-643. URL: http://dx.doi.org/10.1038/ni.2587. doi:10.1038/ni.2587.

55. Chatr-aryamontri, A., Breitkreutz, B.J., Oughtred, R., Boucher, L., Heinicke, S., Chen, D., Stark, C., Breitkreutz, A., Kolas, N., O’Donnell, L., et al. The BioGRID interaction database: 2015 update. Nucleic acids research 2014;:gku1204.

56. Kanehisa, M., Sato, Y., Kawashima, M., Furumichi, M., Tanabe, M.. KEGG as a reference resource for gene and protein annotation. Nucleic Acids Res 2015;URL: http: / / dx. doi . org/10.1093/nar/gkv1070. doi:10.1093/nar/gkv1070.

57. Zhu, F., Shi, L., Engel, J.D., Guan, Y.. Regulatory network inferred using expression data of small sample size: application and validation in erythroid system. Bioinformatics 2015;31(15):2537-2544. URL: http: / / dx.doi.org/10.1093/bioinformatics/btv186.doi:10.1093/bioinformatics/btv186.

58. Park, C.Y., Krishnan, A., Zhu, Q., Wong, A.K., Lee, Y.S., Troyanskaya, O.G.. Tissue-aware data integration approach for the inference of pathway interactions in metazoan organisms. Bioinformatics 2015;31(7):1093-1101. URL: http://dx.doi.org/10.1093/bioinformatics/btu786. doi:10.1093/bioinformatics/btu786.

59. Greene, C.S., Krishnan, A., Wong, A.K., Ricciotti, E., Zelaya, R.A., Himmelstein, D.S., Zhang, R., Hartmann, B.M., Zaslavsky, E., Sealfon, S.C., Chasman, D.I., FitzGerald, G.A., Dolinski, K., Grosser, T., Troyanskaya, O.G.. Understanding multicellular function and disease with human tissue-specific networks. Nat Genet 2015;47(6):569-576. URL: http: / / dx. doi .org/10.1038/ng.3259. doi:10.1038/ng . 3259.

60. Edgar, R., Domrachev, M., Lash, A.E.. Gene expression omnibus: Ncbi gene expression and hybridization array data repository. Nucleic Acids Res 2002;30(1):207-210.

61. Yosef, N., Shalek, A.K., Gaublomme, J.T., Jin, H., Lee, Y., Awasthi, A., Wu, C., Karwacz, K., Xiao, S., Jorgolli, M., Gennert, D., Satija, R., Shakya, A., Lu, D.Y., Trombetta, J.J., Pillai, M.R., Ratcliffe, P.J., Coleman, M.L., Bix, M., Tantin, D., Park, H., Kuchroo, V.K., Regev, A.. Dynamic regulatory network controlling TH17 cell differentiation. Nature 2013;496(7446):461-468. URL: http://dx . doi . org/10 . 1038/nature11981. doi:10.1038/nature11981. 
62. de Jong, H.. Modeling and simulation of genetic regulatory systems: a literature review. J Comput Biol 2002;9(1):67-103. URL: http://dx.doi.org/10.1089/10665270252833208. doi:10.1089/ 10665270252833208.

63. Huynh-Thu, V.A., Sanguinetti, G.. Combining tree-based and dynamical systems for the inference of gene regulatory networks. Bioinformatics 2015;31(10):1614-1622. URL: http://dx.doi.org/10.1093/ bioinformatics/btu863. doi:10.1093/bioinformatics/btu863.

64. Göttgens, B.. Regulatory network control of blood stem cells. Blood 2015;125(17):2614-2620. URL: http: //dx.doi.org/10.1182/blood-2014-08-570226.doi:10.1182/blood-2014-08-570226.

65. Stegle, O., Teichmann, S.A., Marioni, J.C.. Computational and analytical challenges in single-cell transcriptomics. Nat Rev Genet 2015;16(3):133-145. URL: http://dx.doi.org/10.1038/nrg3833. doi:10.1038/nrg3833.

66. Krishnaswamy, S., Spitzer, M.H., Mingueneau, M., Bendall, S.C., Litvin, O., Stone, E., Pe'er, D., Nolan, G.P.. Systems biology. conditional density-based analysis of $\mathrm{t}$ cell signaling in single-cell data. Science 2014;346(6213):1250689. URL: http://dx. doi.org/10.1126/science.1250689. doi:10.1126/science.1250689.

67. Ocone, A., Haghverdi, L., Mueller, N.S., Theis, F.J.. Reconstructing gene regulatory dynamics from highdimensional single-cell snapshot data. Bioinformatics 2015;31(12):i89-i96. URL: http://dx . doi .org/ 10.1093/bioinformatics/btv257. doi:10.1093/bioinformatics/btv257.

68. Chen, H., Guo, J., Mishra, S.K., Robson, P., Niranjan, M., Zheng, J.. Single-cell transcriptional analysis to uncover regulatory circuits driving cell fate decisions in early mouse development. Bioinformatics 2015;31(7):1060-1066. URL: http://dx.doi.org/10.1093/bioinformatics/btu777. doi:10.1093/bioinformatics/btu777.

69. Bendall, S.C., Davis, K.L., Amir, E.a.D., Tadmor, M.D., Simonds, E.F., Chen, T.J., Shenfeld, D.K., Nolan, G.P., Peer, D.. Single-cell trajectory detection uncovers progression and regulatory coordination in human b cell development. Cell 2014;157(3):714-725.

70. Giaever, G., Nislow, C.. The yeast deletion collection: a decade of functional genomics. Genetics 2014;197(2):451-465. URL: http://dx.doi.org/10.1534/genetics.114.161620. doi:10 . 1534/genetics.114.161620. 
71. Schmidt, E.E., Pelz, O., Buhlmann, S., Kerr, G., Horn, T., Boutros, M.. Genomernai: a database for cell-based and in vivo rnai phenotypes, 2013 update. Nucleic acids research 2013;41(D1):D1021-D1026.

72. Yeger-Lotem, E., Riva, L., Su, L.J., Gitler, A.D., Cashikar, A.G., King, O.D., Auluck, P.K., Geddie, M.L., Valastyan, J.S., Karger, D.R., Lindquist, S., Fraenkel, E.. Bridging high-throughput genetic and transcriptional data reveals cellular responses to alpha-synuclein toxicity. Nat Genet 2009;41(3):316-323. URL: http://dx.doi.org/10.1038/ng.337.doi:10.1038/ng.337.

73. Kim, Y.A., Przytycki, J.H., Wuchty, S., Przytycka, T.M.. Modeling information flow in biological networks. Phys Biol 2011;8(3):035012. URL: http://dx.doi.org/10.1088/1478-3975/8/3/ 035012. doi:10.1088/1478-3975/8/3/035012.

74. Huang, S.s.C., Fraenkel, E.. Integrating proteomic, transcriptional, and interactome data reveals hidden components of signaling and regulatory networks. Science Signaling 2009;2(81):ra40-ra40.

75. Yeang, C.H., Ideker, T., Jaakkola, T.. Physical network models. J Comput Biol 2004;11(2-3):243-262.

76. Gitter, A., Bar-Joseph, Z.. Identifying proteins controlling key disease signaling pathways. Bioinformatics 2013;29(13):i227-i236. URL: http://dx . doi org/10.1093/bioinformatics/btt241. doi:10 . $1093 /$ bioinformatics/btt 241

77. Chasman, D., Gancarz, B., Hao, L., Ferris, M., Ahlquist, P., Craven, M.. Inferring host gene subnetworks involved in viral replication. PLoS Comput Biol 2014;10(5):e1003626. URL: http://dx . doi . org/10 . 1371/journal.pcbi.1003626. doi:10.1371/journal.pcbi.1003626.

78. Chasman, D., Ho, Y.H., Berry, D.B., Nemec, C.M., MacGilvray, M.E., Hose, J., Merrill, A.E., Lee, M.V., Will, J.L., Coon, J.J., Ansari, A.Z., Craven, M., Gasch, A.P.. Pathway connectivity and signaling coordination in the yeast stress-activated signaling network. Mol Syst Biol 2014;10:759.

79. Jain, S., Gitter, A., Bar-Joseph, Z.. Multitask learning of signaling and regulatory networks with application to studying human response to flu. PLoS Comput Biol 2014;10(12):e1003943. URL: http: / / dx.doi .org/ 10.1371/journal.pcbi.1003943. doi:10.1371/journal.pcbi.1003943.

80. Gitter, A., Braunstein, A., Pagnani, A., Baldassi, C., Borgs, C., Chayes, J., Zecchina, R., Fraenkel, E.. Sharing information to reconstruct patient-specific pathways in heterogeneous diseases. Pac Symp Biocomput $2014 ;: 39-50$. 
81. Mazza, A., Gat-Viks, I., Farhan, H., Sharan, R.. A minimum-labeling approach for reconstructing protein networks across multiple conditions. Algorithms Mol Biol 2014;9(1):1. URL: http://dx. doi . org/10 . 1186/1748-7188-9-1. doi:10.1186/1748-7188-9-1.

82. Gat-Viks, I., Tanay, A., Raijman, D., Shamir, R.. A probabilistic methodology for integrating knowledge and experiments on biological networks. J Comput Biol 2006;13(2):165-181. URL: http://dx.doi .org/ $10.1089 / \mathrm{cmb} .2006 .13 .165$. doi:10.1089/cmb.2006.13.165.

83. Vaske, C.J., Benz, S.C., Sanborn, J.Z., Earl, D., Szeto, C., Zhu, J., Haussler, D., Stuart, J.M.. Inference of patient-specific pathway activities from multi-dimensional cancer genomics data using paradigm. Bioinformatics 2010;26(12):i237-i245. URL: http://dx.doi.org/10.1093/bioinformatics/btq182. doi:10.1093/bioinformatics/btq182.

84. Wilentzik, R., Gat-Viks, I.. A statistical framework for revealing signaling pathways perturbed by dna variants. Nucleic Acids Res 2015;43(11):e74. URL: http://dx.doi .org/10.1093/nar/gkv203. doi:10.1093/nar/gkv203.

85. Bertrand, D., Chng, K.R., Sherbaf, F.G., Kiesel, A., Chia, B.K.H., Sia, Y.Y., Huang, S.K., Hoon, D.S.B., Liu, E.T., Hillmer, A., Nagarajan, N.. Patient-specific driver gene prediction and risk assessment through integrated network analysis of cancer omics profiles. Nucleic Acids Res 2015;43(7):e44. URL: http: / / dx . doi.org/10.1093/nar/gku1393. doi:10.1093/nar/gku1393.

86. Shrestha, R., Hodzic, E., Yeung, J., Wang, K., Sauerwald, T., Dao, P., Anderson, S., Beltran, H., Rubin, M.A., Collins, C.C., et al. HIT'nDRIVE: Multi-driver gene prioritization based on hitting time. In: Research in Computational Molecular Biology. Springer; 2014:293-306.

87. Vandin, F., Upfal, E., Raphael, B.J.. Algorithms for detecting significantly mutated pathways in cancer. In: Research in Computational Molecular Biology. Springer; 2010:506-521.

88. Kim, Y.A., Cho, D.Y., Dao, P., Przytycka, T.M.. MEMCover: integrated analysis of mutual exclusivity and functional network reveals dysregulated pathways across multiple cancer types. Bioinformatics 2015;31(12):i284-i292. URL: http://dx.doi.org/10.1093/bioinformatics/btv247. doi:10.1093/bioinformatics/btv247.

89. Hormozdiari, F., Penn, O., Borenstein, E., Eichler, E.E.. The discovery of integrated gene networks for autism and related disorders. Genome Res 2015;25(1):142-154. URL: http://dx . doi . org/10.1101/ gr.178855.114. doi:10.1101/gr.178855.114. 
90. Peng, C.H., Jiang, Y.Z., Tai, A.S., Liu, C.B., Peng, S.C., Liao, C.T., Yen, T.C., Hsieh, W.P.. Causal inference of gene regulation with subnetwork assembly from genetical genomics data. Nucleic Acids Res 2014;42(5):2803-2819. URL: http://dx.doi.org/10.1093/nar/gkt1277. doi:10.1093/nar/ gkt 1277 .

91. Bickmore, W.A.. The spatial organization of the human genome. Annual review of genomics and human genetics 2013;14:67-84.

92. Califano, A., Butte, A.J., Friend, S., Ideker, T., Schadt, E.. Leveraging models of cell regulation and GWAS data in integrative network-based association studies. Nature Genetics 2012;44(8):841-847. URL: http://dx.doi.org/10.1038/ng.2355.doi:10.1038/ng.2355.

93. Marbach, D., Lamparter, D., Quon, G., Kellis, M., Kutalik, Z., Bergmann, S.. Tissue-specific regulatory circuits reveal variable modular perturbations across complex diseases. Nat Methods 2016;13(4):366-370. URL: http://dx.doi.org/10.1038/nmeth.3799.doi:10.1038/nmeth.3799.

94. Moreau, Y., Tranchevent, L.C.. Computational tools for prioritizing candidate genes: boosting disease gene discovery. Nat Rev Genet 2012;13(8):523-536. URL: http://dx . doi . org/10 .1038/nrg3253. doi:10.1038/nrg3253.

95. Barabási, A.L., Oltvai, Z.N.. Network biology: understanding the cell's functional organization. Nat Rev Genet 2004;5(2):101-113. URL: http://dx.doi.org/10.1038/nrg1272. doi:10.1038/nrg1272.

96. Köhler, S., Bauer, S., Horn, D., Robinson, P.N.. Walking the interactome for prioritization of candidate disease genes. American journal of human genetics 2008;82(4):949-958. URL: http://dx.doi.org/ $10.1016 / j . a j h g .2008 .02 .013$. doi:10.1016/j.ajhg.2008.02.013.

97. Rackham, O.J., Firas, J., Fang, H., Oates, M.E., Holmes, M.L., Knaupp, A.S., Suzuki, H., Nefzger, C.M., Daub, C.O., Shin, J.W., et al. A predictive computational framework for direct reprogramming between human cell types. Nature genetics 2016;

98. Cahan, P., Li, H., Morris, S.A., Lummertz da Rocha, E., Daley, G.Q., Collins, J.J.. Cellnet: network biology applied to stem cell engineering. Cell 2014;158(4):903-915. URL: http://dx.doi.org/10.1016/j . cell.2014.07.020.doi:10.1016/j.cell.2014.07.020.

99. Buenrostro, J.D., Wu, B., Litzenburger, U.M., Ruff, D., Gonzales, M.L., Snyder, M.P., Chang, H.Y., 
Network terminology. A molecular network is defined by the type of entities it connects and the information it contains about those connections.

A. Network elements. Nodes in a molecular network are intracellular molecules, frequently genes or proteins. Edges represent interactions (binding, regulatory, post-translational modification, correlation) and may be directed (if it is known that the first molecule's activity or function influences the second's) or undirected.

B. Network structure and function. On the right is a cartoon showing a kinase $g_{1}$ and transcription factor (TF) $g_{2}$ whose coordinate activity regulates a gene $g_{3}$. The structure shows $g_{1}$ and $g_{2}$ as regulators of $g_{3}$, and the function $f$ quantifies the relationship between regulator state and target. This function estimates the state or activity level of the target gene in one or multiple time points/conditions as a function of state/activity of regulators, or other input parameters. In the literature, this function has taken many forms, including simple linear regression, linear or non-linear differential equation, and probabilistic Boolean function.

C. Types of networks. Regulatory networks describe transcriptional regulatory programs (as in Panel B). Signaling networks describe cascades of post-translational modifications from signaling receptors to transcription factors.

Functional networks are undirected, and represent statistical dependencies between nodes. Physical networks represent experimentally observed binding interactions between proteins or between proteins and DNA or RNA. In any type of network, weights on edges may correspond to statistical significance, confidence, or inferred importance of the edge.

D. Organizational units. Networks are composed of smaller structural units. A subnetwork is a subset of nodes and edges from the original network; subnetworks are used to predict functional coordination between nodes. A regulatory path is a chain of nodes and edges that posits the ordered mechanism by which upstream regulators and transcription factors influence the regulation of a target gene. A regulatory module is a group of genes that are co-expressed and are predicted to share the same regulatory program. A hub node has a high degree (number of neighbors); hubs are often predicted to be important regulators of one or more cellular pathways.

Box 1: Network terminology

Figure 1: Overview of major computational network biology tasks addressed here. Shown are the key inputs, algorithmic approaches and outputs produced in each type of task. (A) Network reconstruction for identifying a single network or multiple context-specific networks. (B) Network-based interpretation to identify a relevant subnetwork that integrates diverse data types (e.g. mRNA and proteomic measurements) on a skeleton network. (C) Network-based interpretation of genetic variants to identify either subnetworks or network modules. (D) Network-based prioritization to prioritize genes for further experiments based on an input gene set and measured expression levels and proximity of candidate genes on the skeleton graph.

Greenleaf, W.J.. Single-cell chromatin accessibility reveals principles of regulatory variation. Nature 2015;523(7561):486-490.

100. Gilbert, L.A., Horlbeck, M.A., Adamson, B., Villalta, J.E., Chen, Y., Whitehead, E.H., Guimaraes, C., Panning, B., Ploegh, H.L., Bassik, M.C., Qi, L.S., Kampmann, M., Weissman, J.S.. Genome-scale crisprmediated control of gene repression and activation. Cell 2014;159(3):647-661. URL: http://dx.doi . org/10.1016/j.cell.2014.09.029. doi:10.1016/j.cell.2014.09.029.

101. Wang, T., Wei, J.J., Sabatini, D.M., Lander, E.S.. Genetic screens in human cells using the crispr-cas9 system. Science 2014;343(6166):80-84. URL: http://dx.doi.org/10.1126/science.1246981. doi:10.1126/science.1246981. 


Problem
A. Reconstruct
networks
(one or more
contexts)

\section{B. Network-}

based

interpretation

$x^{2}$

C. Interpret

genetic variants

in living systems

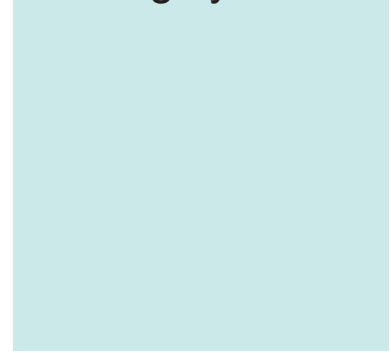

D. Prioritize

genes for

experiments

\section{Given}

Expression levels

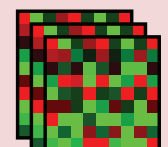

mRNAs

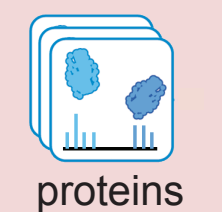

(some methods)

Prior network

(some methods)

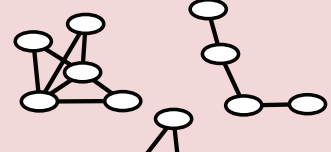

वी

(motif, ChIP, PPI)

Measurements Skeleton network

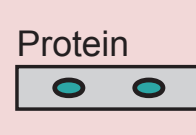

mRNA

무

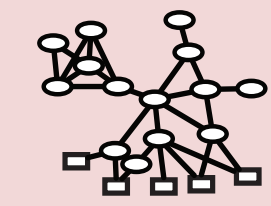

口iำ

OProtein
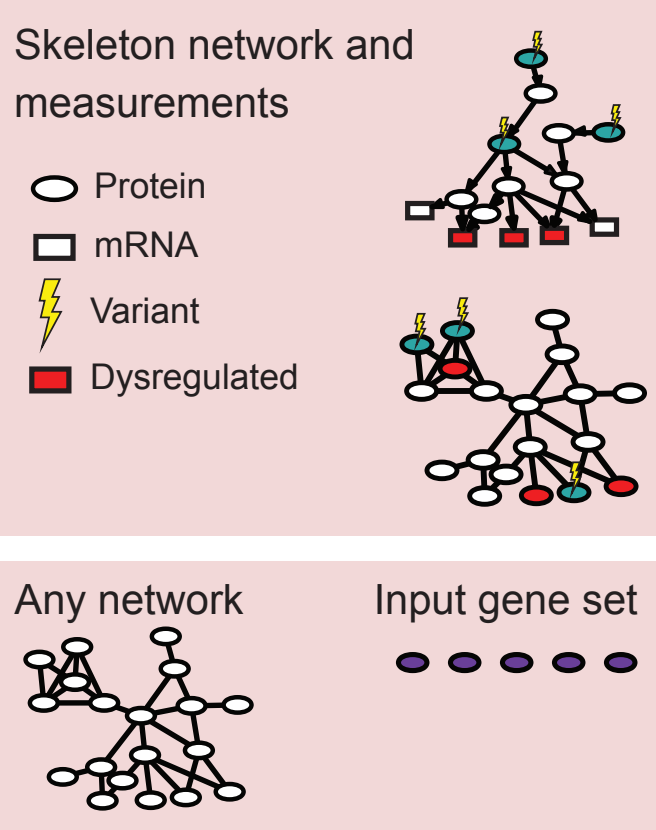

Input gene set

00000
Do

- Sparse regression

- Random Forests

- Graphical models

- Causal networks

- Sparse inverse

covariance selection

Share data between

cell line/tissue

based on hierarchy

- Multi-task learning

- Steiner trees

- Maximum flow

- Path-based

methods

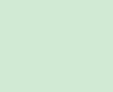

\section{- Test associations \\ between candidate \\ variants, condition, \\ targets \\ - Information flow \\ - Module finding}

\section{Score genes:}

- Distance to all input

genes

- Expected expression

outcome

\section{Output}

Regulatory network

$\begin{array}{ccc}\text { Structure } & & \text { Function } \\ \text { Regulators } g_{1} & g_{2} & \\ \text { Target } & g_{g_{3}} & \\ g_{3}=f\left(g_{1}, g_{2}\right) & \end{array}$

Functional network

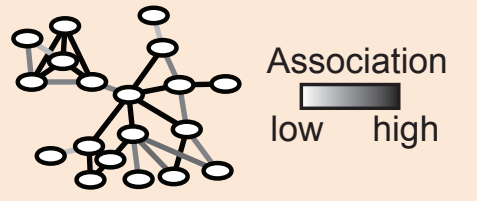

Context-specific network

Context A only Context B only

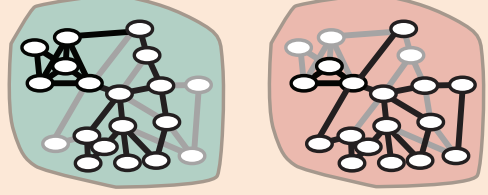

Relevant subnetwork
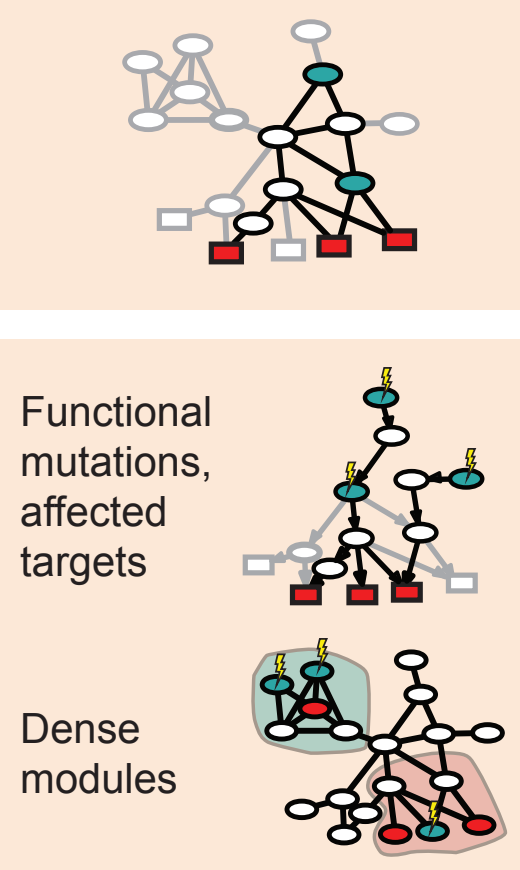

Scores for genes

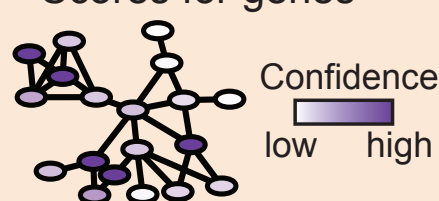




\section{Box 1}

A.

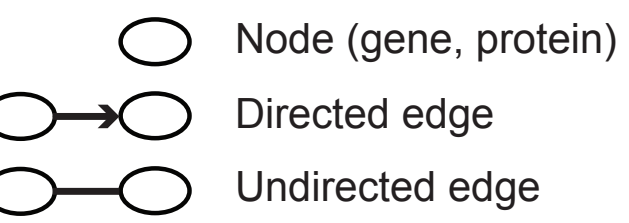

C.

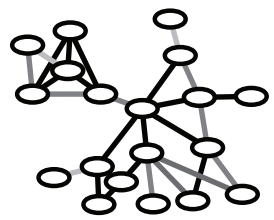

Undirected

- Functional

- Physical
B.

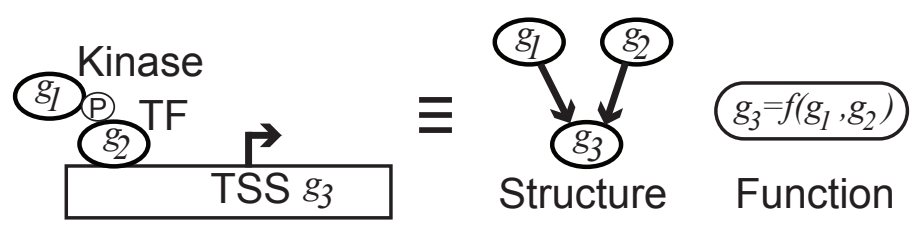

D.

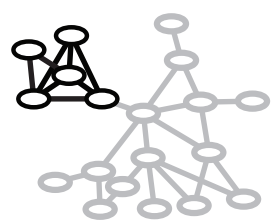

Subnetwork

(black: included; grey: not included)
O Upstream regulator

Transcription factor Target

Regulatory path

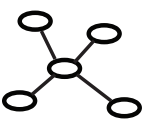
Hub node (many neighbors)

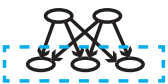

Regulatory module (targets in box) 
graphical abstract

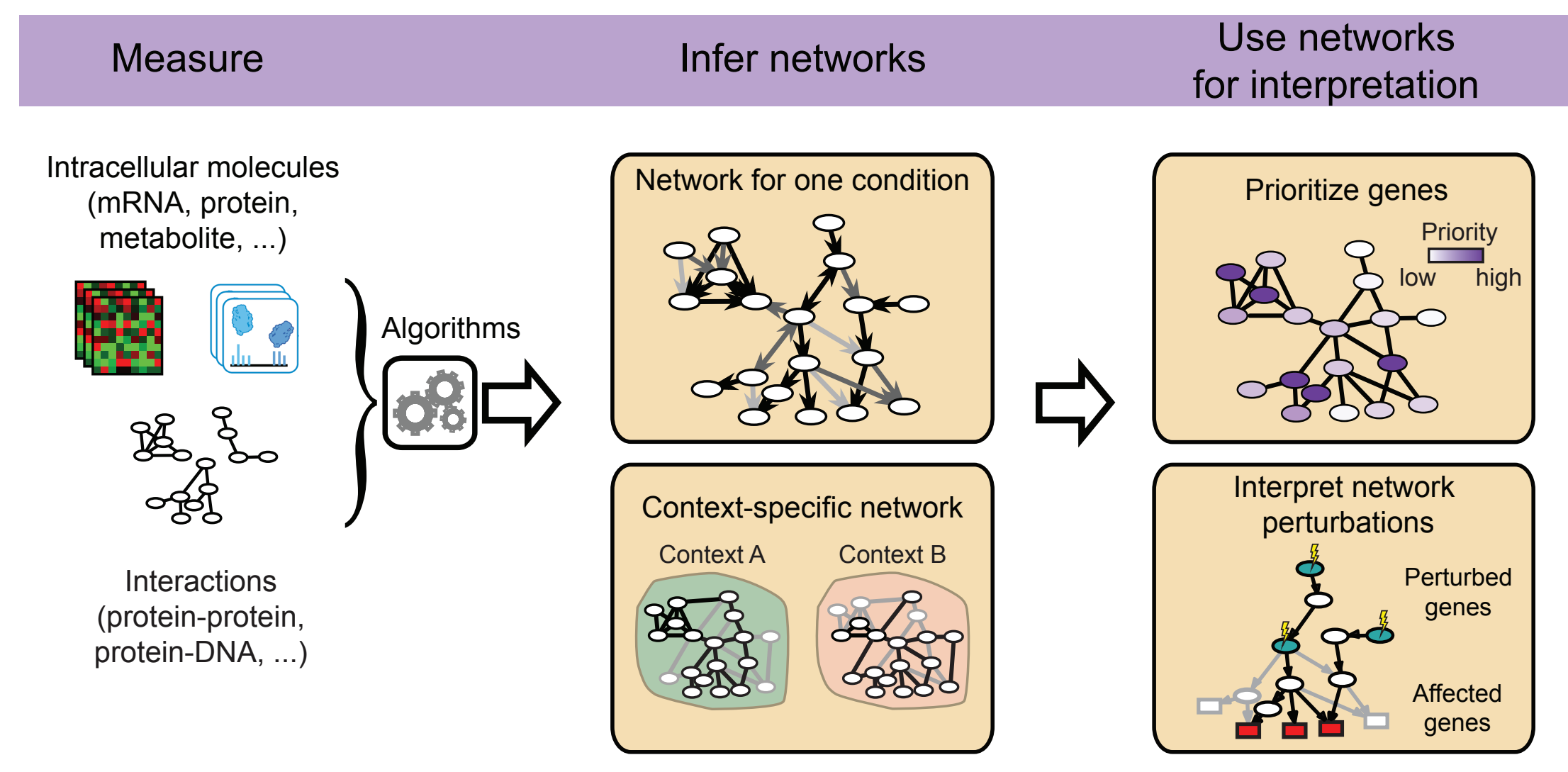

